\title{
Status of CHEK2 and p53 in patients with early-onset and conventional gastric cancer
}

\author{
JULITA MACHLOWSKA ${ }^{1,2}$, PRZEMYSŁAW KAPUSTA ${ }^{1}$, MAŁGORZATA SZLENDAK ${ }^{2,3}$, \\ ANNA BOGDALI $^{1}$, FOLKERT MORSINK ${ }^{4}$, PAWEŁ WOŁKOW ${ }^{1}$, RYSZARD MACIEJEWSKI ${ }^{2}$, \\ G. JOHAN A. OFFERHAUS ${ }^{2,4}$ and ROBERT SITARZ $Z^{2,4,5}$ \\ ${ }^{1}$ Center for Medical Genomics OMICRON, Jagiellonian University Medical College, 31-034 Kraków; \\ Departments of ${ }^{2}$ Human Anatomy and ${ }^{3}$ Surgical Oncology, Medical University of Lublin, 20-090 Lublin, \\ Poland; ${ }^{4}$ Department of Pathology, University Medical Center Utrecht, 3584 CX Utrecht, The Netherlands; \\ ${ }^{5}$ Department of Surgery, Center of Oncology of The Lublin Region St. Jana z Dukli, 20-090 Lublin, Poland
}

Received August 7, 2020; Accepted February 8, 2021

DOI: 10.3892/ol.2021.12609

\begin{abstract}
Gastric cancer (GC) is the fourth most common cause of cancer-associated death. Based on the age at diagnosis, GC is divided into early-onset GC (EOGC; $\leq 45$ years) and conventional GC (CGC; $>45$ years). Mutations in the cell cycle checkpoint kinase 2 (CHEK2) and TP53 genes are associated with several types of cancer; however, their genetic defects in GC remain poorly understood. The aim of the present study was to determine the subcellular distribution of the CHEK2 protein and its redistribution following DNA damage, to improve the understanding of the DNA damage response. Genetic alterations and patterns of expression of CHEK2 and p53 proteins were investigated to identify potential biological markers and indicators of GC development. Additionally, the affected signaling pathways and their clinical importance in GC development and associated syndromes were investigated. A total of 196 GC samples (89 CGC and 107 EOGC samples) were used in the present study. DNA from 53 samples (18 CGC and 35 EOGC samples) was sequenced using targeted next-generation sequencing technology to identify and compare common and rare mutations associated with GC. Subsequently, the cytoplasmic and nuclear expression levels of CHEK2, phosphorylated (p)-CHEK2 at threonine 68 and p53 in GC tissues were determined via immunohistochemistry. Sequencing resulted in the identification of 63 single nucleotide polymorphisms (SNPs) in the CHEK2 gene amongst 5 different variants, and the intron variant c. $319+379 \mathrm{~A}>\mathrm{G}$ was the most common SNP. In the TP53 gene, 57 different alterations were
\end{abstract}

Correspondence to: Dr Robert Sitarz, Department of Human Anatomy, Medical University of Lublin, 4 Jaczewskiego Street, 20-090 Lublin, Poland

E-mail: r.sitarz@umlub.pl

Key words: gastric cancer, early-onset subtype, checkpoint kinase 2, p53, immunohistochemistry, next-generation sequencing detected amongst 9 variant types, and the missense variant c. $215 \mathrm{C}>\mathrm{G}$ was the most common. Nuclear CHEK2 expression was high in both the EOGC and CGC subtypes. However, the prevalence of cytoplasmic CHEK2 expression $(\mathrm{P}<0.001)$ and nuclear $\mathrm{p}-\mathrm{CHEK} 2$ expression $(\mathrm{P}=0.011)$ was significantly higher in CGC compared with in EOGC tissues. There was a statistically significant difference between high and low cytoplasmic CHEK2 expression in patients with p53-positive EOGC compared with in patients with p53-positive CGC $(\mathrm{P}=0.002)$. The present study was designed to determine the association between $\mathrm{CHEK} 2$ and p53 expression patterns in patients with EOGC and CGC, as well as genetic alterations in the CHEK2 and TP53 genes.

\section{Introduction}

Gastric cancer (GC) is the fourth most common cause of cancer-related death worldwide (1). The incidence of GC increases with age, and the median age at diagnosis is 70 years (2). However, $\sim 10 \%$ of the gastric carcinomas are detected at the age of 45 or younger (3). GC is a multifactorial disease, and both environmental and genetic factors serve a role in its etiology (2-4). GC in younger patients is termed early-onset gastric cancer (EOGC), and is likely caused by a predisposing genotype that facilitates the development of the cancer due to various environmental triggers (4). It has been postulated that genetic factors may be more important in EOGC than in elder patients, as younger patients have a shorter duration of exposure to environmental carcinogens (3).

Currently, there are two distinguished classifications of GC, the World Health Organization (WHO) and the Laurén classification system. According to the Laurén classification, which is often used for diversification, there are two histological subtypes: Diffuse and intestinal (5). Intestinal gastric carcinoma is more common in elder patients and it is frequently accompanied by multifocal atrophic gastritis. This is followed by intestinal metaplasia and leads to cancer via dysplasia. Younger patients more commonly exhibit a diffuse cancer, in which hereditary factors serve a major role in the development. 
DNA damage response genes are involved in the preservation of a healthy genome. Defects in cell cycle checkpoints and DNA repair genes, such as ATM, CHEK2, BRCA1,BRCA2 and $T P 53$, particularly mutations and/or aberrant downregulation, may be associated with the development of gastric cancer (6).

The cell cycle checkpoint kinase 2 (CHEK2) gene serves a significant role in the DNA damage response signaling network, particularly in cancer development (7). Germline mutations in the gene have been reported in families of patients with cancer; particularly for breast cancer (8-10) and prostate cancer $(11,12)$, but also in other types of cancer $(7,13,14)$, including gastric cancer (15). The most prevalent mutant alleles of $C H E K 2$ are three mutant alleles resulting in protein truncation (1100delC, IVS2G $>$ A, del5395), one which is a missense variant (I157T) (16).

In humans, the CHEK2 protein is a $65 \mathrm{kDa}$ polypeptide that consists of 543 amino acids and three different functional domains. The $\mathrm{N}$ terminal SQ/TQ cluster domain contains a region rich in serine-glutamine and threonine-glutamine motifs, which are potential sites of phosphorylation by ATR and ATM kinases (part of the PI3K family) (17). Under physiological conditions, CHEK2 in retained in the nucleus in an inactive, monomeric form. DNA damage initiates phosphorylation at Thr68 and other sites of the SQ/TQ cluster domain. The phosphorylated site binds to the forkhead-associated domain and forms a dimer that is further activated by autophosphorylation at the C-terminal serine/threonine kinase domain (18). As a result, the dimer dissociates into fully active monomers. The Thr68 phosphorylated form of CHEK 2 creates distinct nuclear foci as a reaction to ionizing radiation (19). Only the activated form of CHEK2 is present at sites of DNA double stranded breaks. These observations suggest that CHEK2 is regulated at the site of the DNA double stranded break, and that phosphorylated $\mathrm{CHEK} 2$ has an important role in the DNA damage response. Our current study is focused on both forms of CHEK2 to examine their role in the development of age-dependent subtypes of gastric carcinoma.

The TP53 gene is located on the short arm of chromosome 17 (17p13.1) (18). Multiple studies have demonstrated the genetic association between TP53 alterations and cancer susceptibility. The TP53 gene is the most frequently mutated gene $(>50 \%)$ in human cancer, suggesting that the TP53 gene serves a pivotal role in preventing the formation of cancer (19). In gastric carcinoma the mutations are located broadly across a gene, from exons 4-11 with hot spots of mutations at codons 175, 248, 273, 282, 245 and 213. Additionally, G:C>A:T transitions at $\mathrm{CpG}$ sites are the most popular types of mutation (20).

The p53 protein serves an important role in regulation or cancer progression through the regulation of apoptosis, the cell cycle and genomic stability (21). p53 can stimulate DNA repair proteins when DNA damage is persistent or serious. It can prevent proliferation by arresting the cell cycle at the G1/S regulation point following recognition of DNA damage. When a cell is arrested at this checkpoint, the DNA repair proteins attempt a repair. However, if the cells are arrested at this checkpoint for a sufficient amount of time, the cell may instead undergo apoptosis (22).

The present study focused on the frequency of genetic alterations in the CHEK2 and TP53 genes, and the expression patterns of the phosphorylated and non-phosphorylated
CHEK2 and p53 proteins in both conventional (C)GC and early onset (EO)GC, to assess the effects of CHEK2 and p53 in the development of the heterogeneous nature of GC.

\section{Materials and methods}

Patients. The tissue samples used in the present study included 89 cases of CGC, diagnosed between 1993-2003 and 107 samples of EOGC, obtained from the Academic Medical Centre in Amsterdam as well as collected from 24 institutions in the Netherlands, Finland and Poland. Tumor samples were classified according using the Laurén classification system (23) as either intestinal or diffuse gastric adenocarcinomas (Table I). The tissues were previously used for a previous analysis (24), where immunohistochemical labelling was performed to determine the expression patterns of several gastric cancer markers amongst EOGC and CGC. For sequencing analysis, $53 \mathrm{GC}$ tissues were selected, 35 with EOGC and 18 with CGC, taking into consideration the DNA quality status of the samples. The present study was approved by the Medical University of Lublin Bioethical Committee and was performed in accordance with the Declaration of Helsinki.

DNA extraction. Tissues were obtained as previously described by Sitarz et al (25). Genomic DNA extraction from formalin-fixed paraffin-embedded (FFPE) tissues was performed using the QIAamp DNA Mini kit (Qiagen $\mathrm{GmbH}$ ) or a Puregene DNA Isolation kit (Gentra, Inc.) in accordance with the manufacturers' protocols. DNA was quantified using a Quantus $^{\mathrm{TM}}$ Fluorometer with the QuantiFluor ${ }^{\circledR}$ dsDNA system according to the manufacturer's protocol (Promega Corporation). DNA quality was assessed using an Agilent 2200 TapeStation and Genomic DNA ScreenTape (Agilent Technologies, Inc.). Further evaluation of the suitability of the samples for sequencing was performed using an Infinium HD FFPE QC assay protocol (Illumina, Inc.). The Real-Time PCR using a CFX96 Touch $^{\mathrm{TM}}$ Real-Time PCR Detection system (Bio-Rad Laboratories, Inc.) was used to assess the amplification status of each sample.

\section{Library preparation using a target enrichment workflow for} next generation sequencing. Libraries for the sequencing experiments were prepared using a Trusight Rapid Capture Preparation kit (Illumina, Inc.) according to the manufacturer's protocol. DNA samples were standardized to $50 \mathrm{ng}$ and underwent a tagmentation step. The produced libraries from input of genomic DNA were amplified and adapter tagged multiple short library fragments of 220-350 base pairs were prepared. Libraries for each sample were combined to ensure proper complexity for a single run. Biotin labelled probes were used to target regions of interest. Further hybridization steps were performed using custom synthesized oligos that captured genes with an assigned predisposition towards multiple cancer types. The sequencing panel, TruSight Cancer Panel (Illumina, Inc.), targeted 94 genes and 284 SNPs linked to common and rare cancers. The complete list of target genes and SNPs is available from illumina.com/techniques/popularapplications/genotyping.html. Standard pools were enriched with regions of interest through streptavidin coated beads that 
Table I. Histological types of GC samples analyzed in the study $(\mathrm{n}=196)$.

\begin{tabular}{lcc}
\hline Characteristic & $\begin{array}{c}\text { Early-onset GC } \\
(\mathrm{n}=107)\end{array}$ & $\begin{array}{c}\text { Conventional GC } \\
(\mathrm{n}=89)\end{array}$ \\
\hline Age (range), years & $\leq 45(21-45)$ & $>45(47-86)$ \\
Histology, $\mathrm{n}(\%)$ & & $42(47)$ \\
Intestinal & $21(20)$ & $37(42)$ \\
Diffuse & $78(73)$ & $10(11)$ \\
Mixed & $8(7)$ & \\
\hline
\end{tabular}

$\mathrm{GC}$, gastric cancer.

bound to the biotinylated probes. Then, DNA fragments were eluted from the beads and another round of hybridization and enrichment was performed. The final amplified post-capture library concentrations were assessed using a Quantus ${ }^{\mathrm{TM}}$ Fluorometer according to the manufacturer's protocol. Libraries were quantified by qPCR using a KAPA Library Quantification kit (KAPA Biosystems, Inc.). The size of the obtained libraries fragments was evaluated using an Agilent 2200 TapeStation and High Sensitivity D1000 ScreenTape system (Agilent Technologies, Inc.). Post capture enriched libraries were sequenced on a MiSeq Sequencing Platform (Illumina, Inc.) using the manufacturer's workflow. The concentration of loaded libraries amounted to $10 \mathrm{pM}$. The sequencing experiments were performed using a MiSeq Reagent kit version 2 (300 cycles).

Data processing. Results from each sample were mapped to the human reference genome GRCh37, also known as hg19 (Human Genome version 19) using Burrows-Wheeler Aligner (BWA-mem, version 0.7.5). The reads with low mapping quality scores, unmapped reads and duplicates were filtered out using Samtools version 0.1.19 (26). Local realignment of reads around indels (insertion or deletion) and detection of systematic errors in base quality scores were performed using Genome Analysis Toolkit (27). The reads mapped outside the target region were removed. Variant calling for germline SNPs and indels was performed using the GATK HaplotypeCaller tool (28). The call sets of SNPs and small insertions and deletions were separated for further filtering. Hard filters applied to variant call sets for SNPs were, QualByDepth (QD)<2.0, RMSMappingQuality (MQ)<40.0, FisherStrand (FS) $>60.0$, HaplotypeScore $>13.0$, QRankSum $<-12.5$ and ReadPosRankSum<-8.0; and for INDELs, QD $<2.0$, ReadPosRankSum<-20.0, FS>200.0. Filtered variants were concatenated into one record (VCF file) and the discovered variants were annotated with SnpEff (version 4.2) using GEnome MINIng (Gemini) version 0.18.3, and loaded into an SQLite database (29).

Immunohistochemistry. CHEK2 immunohistochemical staining was performed using a mouse monoclonal antibody for CHEK2 (Santa Cruz Biotechnology, Inc.; cat. no. sc5278; 1:200; incubation period $1 \mathrm{~h}$ ), a rabbit monoclonal antibody against p-CHEK2 (Thr68; Cell Signaling Technology,
Inc.; cat. no. 2197; 1:50; ARS pH 9; $72 \mathrm{~h}$ incubation at $4^{\circ} \mathrm{C}$ ). The sections $(4 \mu \mathrm{m})$ were deparaffinized and endogenous peroxidase activity was blocked by immersion in $0.3 \% \mathrm{H}_{2} \mathrm{O}_{2}$ and in methanol for $20 \mathrm{~min}$. Antigen retrieval was performed in sodium citrate/ARS buffer $(0.01 \mathrm{M} / \mathrm{pH} 6.0)$ for $10 \mathrm{~min}$ at $120^{\circ} \mathrm{C}$ or in Tris/EDTA buffer $(10 \mathrm{mM} / 1 \mathrm{mM}$; pH 9.0) for $10 \mathrm{~min}$ at $120^{\circ} \mathrm{C}$. Subsequently, once the samples had cooled for $10 \mathrm{~min}$, they were washed in PBS and blocked using PBS with 5\% normal goat serum. Then the material was incubated with the antibodies. Antibody binding was visualized using a Powervision + poly-HRP detection system (Leica Microsystems; cat. no. PV6107) with Bright DAB (Immunologic; cat. no. BS04-110) as the chromogen for CHEK2, and PowerDAB (Immunologic; cat. no. BS03-25) used as the chromogen for p-CHEK2. Sections were counterstained using hematoxylin.

TP53 staining; sections (4- $\mu \mathrm{m}$ thick) were deparaffinized and antigen retrieval was performed by boiling in $10 \mathrm{mM}$ Tris/1 mM EDTA (pH 9.0) for $10 \mathrm{~min}$. Subsequently, slides were immersed in $0.3 \%$ hydrogen peroxide in methanol for 30 min and nonspecific binding was blocked with 5\% normal goat serum for $1 \mathrm{~h}$ at room temperature. The sections were incubated for $1 \mathrm{~h}$ at room temperature with an anti-p53 antibody (monoclonal antibody combination of DO-7 and BP53-12; Neomarkers, Inc.; 1:2,000). The Ultravision anti-polyvalent HRP detection system (Lab Vision Corp.) was used to visualize antibody binding with 3,30-diaminobenzidine used as the chromogen. Sections were counterstained using hematoxylin.

Scoring. Scoring of immunohistochemistry was performed for analysis of CHEK 2 and p53 expression. Scoring was performed independently by two researchers, any disagreements in score were reanalyzed by the expert using a multiheaded microscopes in accordance with his judgement. Cases where the staining was not clearly abnormal were categorized into the normal group. No slides exhibited increased negative staining around the edges, and the age of the block had no impact on immunohistochemical staining. Multiple scoring systems in the literature were considered to ensure a system that adequately reflected the results generated was used, and all cases were re-examined based on the agreed upon scoring parameters.

Immunohistochemical labelling revealed the patterns of CHEK2 and p-CHEK2 expression in GC (Fig. 1). The scoring criteria for the quantity of the tumor cells were: 1 , No staining; 2 , weak staining ( $\leq 10 \%$ of the cancer cells); 3 , moderate staining (10-50\% of the cancer cells); 4 , strong staining (nuclear staining in $>50 \%$ of the cancer cells). For statistical analysis, scores 0 and 1 were categorized as 'CHEK2 low staining' and scores 2 and 3 as 'CHEK2 high staining'. The following scoring system was used for p53: Cases scored as positive showed strong nuclear staining in $>30 \%$ of cells, whereas normal mucosa showed scant staining in the proliferative zone only.

Statistical analysis. Statistical analysis (including immunohistochemistry staining of CHEK 2 and p-CHEK2 proteins, immunohistochemical staining of p53 and association between the genetic alterations of CHEK2 and TP53 genes and their 


\section{CHEK-2}

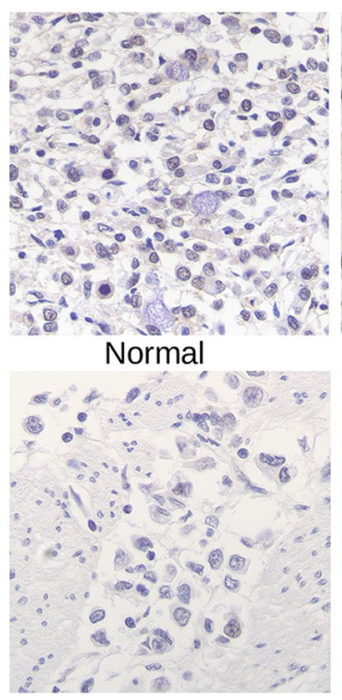

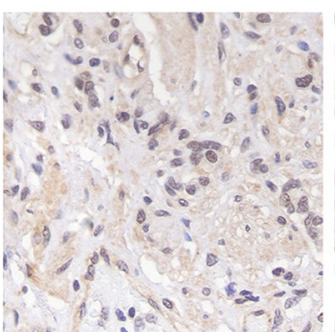

$0+$

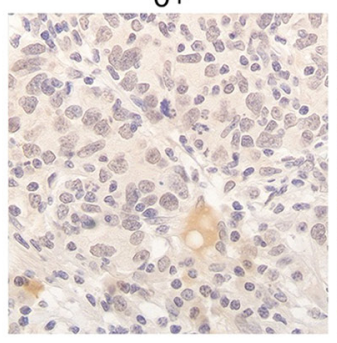

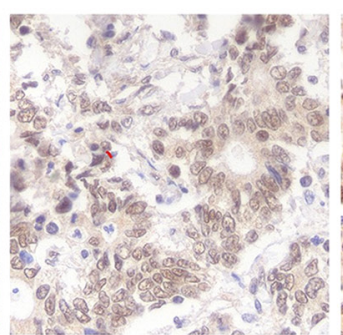

$1+$

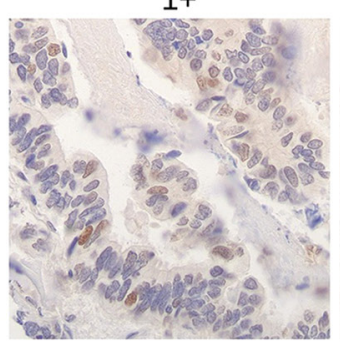

p-CHEK2

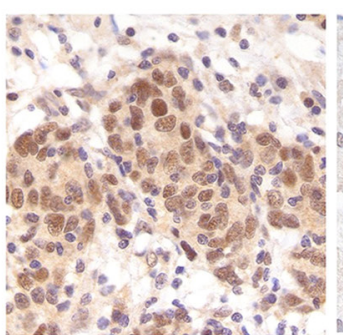

$2+$
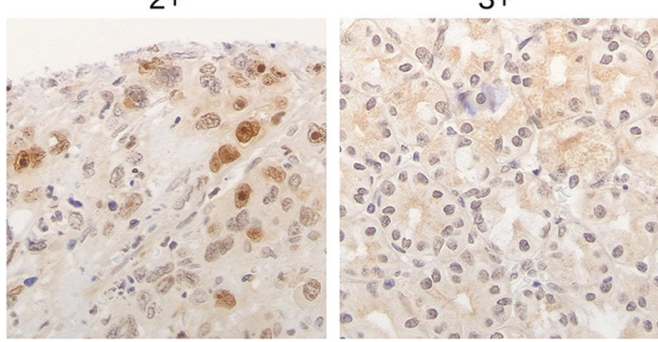

$3+$
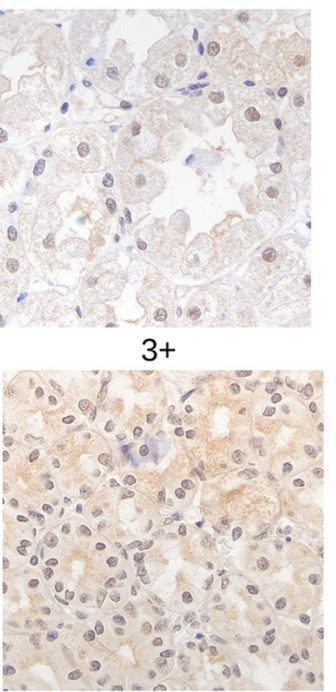

Figure 1. Immunohistochemical staining of CHEK2 and p-CHEK2 in gastric cancer tissues (magnification, x100). The following scoring criteria of the tumor cells were used: $0+$, no staining; $1+$, weak diffuse cytoplasmic staining (may contain stronger intensity in $<10 \%$ of the cancer cells); $2+$, moderate to strong cytoplasmic staining in $10-50 \%$ of the cells; $3+,>50 \%$ of the tumor cells stained with strong intensity. p-CHEK 2 , phosphorylated checkpoint kinase 2 .

protein expression levels) were performed using the $\chi^{2}$ test. For this purpose we used GraphPad programme and applied the $2 \times 2$ contingency table. We chose chi-square test with the Yates' continuity correction, which is designed to make the chi-square approximation better. We calculated two-tailed (also called two-sided) $\mathrm{P}$-values. $\mathrm{P}<0.05$ was considered to indicate a statistically significant difference.

\section{Results}

CHEK2 genetic alterations amongst EOGC and CGC. Next-generation sequencing results were generated for 53 patients with GC, 35 patients with EOGC and 18 patients with CGC. DNA from all samples was successfully amplified, libraries were constructed and a minimum coverage amongst regions of interest was obtained in all tested samples. Results from next-generation targeted sequencing for $C H E K 2$ are shown in Table II. A total of 5 different variants in CHEK2 were found amongst 53 cases: 2 missense variants, 2 synonymous variants and 1 intron alteration. The c.1741G $>$ T missense alteration in $C H E K 2$ was observed only in one case and was described as a medium impact mutation, according to Gemini. This variant seemed to be very rare, as it had not been previously described in CHEK2 and has never been reported in NCBI ClinVar or dbSNP (Table SI). Both missense variants: c. $1246 \mathrm{~A}>\mathrm{G}$ (medium) and the synonymous variant: c.1245C $>\mathrm{T}$ (low) were detected in 5 out of 53 cases. The $C H E K 2$ c.252A $>\mathrm{G}$ synonymous variant, which according to NCBI ClinVar database, is associated with a hereditary predisposition to colorectal cancer and familial breast cancer, was detected in 5 cases. Interestingly the intron variant c. $319+379 A>G$ was detected in 47 cases, but according to Gemini, its impact was low. The detected mutations are fairly uncommon for gastric carcinoma; they were not found in Catalogue of Somatic Mutations in Cancer (COSMIC).
TP53 genetic alterations amongst EOGC and CGC. Results from next-generation sequencing for TP53 are shown in Table III. A total of 9 variant alterations in TP53 were detected in 53 patients: 5 missense variants, 2 synonymous variants, 1 frameshift variant and 1 premature stop codon. The c.1129A >C missense variant was found in one case (EOGC, 1 HET-heterozygote) and was described as a medium impact mutation, according to Gemini (Table SII), and present in dbSNP and ClinVar where it was described to be associated with Li-Fraumeni syndrome, but not present in the COSMIC database. The c.1014C $>\mathrm{T}$ synonymous variant was present in 1 EOGC case as heterozygous, was considered a low impact alteration, described in clinical repositories as related to hereditary cancer predisposing syndrome and Li-Fraumeni syndrome, and in COSMIC it was described as being related to skin and upper aerodigestive tract. A deletion (frameshift variant): c.851_852delCA was observed in one CGC case, described as high impact, and was considered as rare as it was not described in any of the databases. The missense alteration c.844C $>\mathrm{G}$ was reported in 1 EOGC case, as heterozygous, was considered medium impact and was associated with hereditary cancer predisposing syndrome, and in COSMIC, it was described as being associated with lung and breast cancer, as well as hematopoietic and lymphoid, upper aerodigestive tract and pancreatic neoplastic disease. This alteration was also found in stomach cancer by Rugge et al (30) and by $\mathrm{Xu}$ et al (31).

Missense variant c. $818 \mathrm{G}>\mathrm{A}$ was found in one patient with $\mathrm{CGC}$, as heterozygous, medium impact, related to Li-Fraumeni syndrome, thyroid cancer and hereditary cancer predisposing syndrome according to the ClinVar database; and according to COSMIC, it was found in multiple studies in stomach cancer, but predominantly in large intestine, breast, lung, central nervous system and ovary cancer. Variant c.734G $>$ A was a missense alteration, found in one 
Table II. Presentation of variants identified in CHEK2 gene in EOGC $(n=35)$ and CGC $(n=18)$ cases.

\begin{tabular}{lllccc}
\hline Variant type & Protein change & Molecular consequence & Exon & CGC mutations & EOGC mutations \\
\hline c. $1741 \mathrm{G}>\mathrm{T}$ & p. Val581Leu & Missense variant & 16 & - & 1 HET \\
c.1246A $>$ G & p. Lys416Glu & Missense variant & 12 & 3 HET & 2 HET \\
c.1245C $>$ T & p. Ser415Ser & Synonymous variant & 12 & 3 HET & 2 HET \\
c. $252 \mathrm{~A}>\mathrm{G}$ & p. Glu84Glu & Synonymous variant & 2 & 3 HET & 2 HET \\
c. $319+379 \mathrm{~A}>\mathrm{G}$ & - & Intron variant & 2 & 7 HET + 10 ALT & 12 HET + 18 ALT
\end{tabular}

HET, heterozygous; ALT, alternative homozygous (identical mutation of both paternal and maternal alleles); CHEK2, checkpoint kinase 2; GC, gastric cancer; EOGC, early-onset GC; CGC, conventional GC.

Table III. Presentation of variants detected in TP53 gene in EOGC $(n=35)$ and CGC $(n=18)$ cases.

\begin{tabular}{|c|c|c|c|c|c|}
\hline Variant type & Protein change & Molecular consequence & Exon & CGC mutations & EOGC mutations \\
\hline c. $1129 \mathrm{~A}>\mathrm{C}$ & p. Thr377Pro & Missense variant & 11 & - & $1 \mathrm{HET}$ \\
\hline c. $1014 \mathrm{C}>\mathrm{T}$ & p. Phe338Phe & Synonymous variant & 10 & - & $1 \mathrm{HET}$ \\
\hline c.851_852delCA & p. Thr284fs & Frameshift variant & 8 & $1 \mathrm{DEL}$ & - \\
\hline c. $844 \mathrm{C}>\mathrm{G}$ & p. Arg282Gly & Missense variant & 8 & - & $1 \mathrm{HET}$ \\
\hline c. $818 \mathrm{G}>\mathrm{A}$ & p. Arg273His & Missense variant & 8 & $1 \mathrm{HET}$ & - \\
\hline c. $734 \mathrm{G}>\mathrm{A}$ & p. Gly245Asp & Missense variant & 7 & $1 \mathrm{HET}$ & - \\
\hline c. $639 \mathrm{~A}>\mathrm{G}$ & p. Arg213Arg & Synonymous variant & 6 & $3 \mathrm{HET}$ & $1 \mathrm{HET}$ \\
\hline c. $.586 \mathrm{C}>\mathrm{T}$ & p. $\operatorname{Arg} 196 *$ & Nonsense variant & 6 & $1 \mathrm{HET}$ & - \\
\hline c. $215 \mathrm{C}>\mathrm{G}$ & p. Pro72Arg & Missense variant & 4 & $11 \mathrm{HET}+6 \mathrm{ALT}$ & $12 \mathrm{HET}+17 \mathrm{ALT}$ \\
\hline
\end{tabular}

HET, heterozygous; ALT, alternative homozygous (identical mutation of both paternal and maternal alleles); DEL, deletion; GC, gastric cancer; EOGC, early-onset GC; CGC, conventional GC.

case (CGC, 1 HET) and was described as a medium impact mutation, described in dbSNP and ClinVar as associated with Li-Fraumeni syndrome and hereditary cancer predisposing syndrome. In the COSMIC database, it was shown to be found in large intestine, esophagus, hematopoietic and lymphoid, lung and breast neoplasms. In cancers of the stomach, the variant was previously reported by Gleeson et al (32) and Kim et al (33).

Interestingly, variant c.639A $>\mathrm{G}$ (synonymous) was found in 3 CGC cases and 1 EOGC case as heterozygous. This was a low impact variant, associated with hereditary cancer predisposing syndrome and Li-Fraumeni syndrome, and was not found in stomach cancer previously according to COSMIC. However it had been detected in large intestine, soft tissue and central nervous system neoplasms. The variant causing an introduction of premature stop codon, c.586C $>\mathrm{T}$ was considered high impact and was detected in one CGC case (HET), associated with hereditary cancer predisposing syndrome and Li-Fraumeni syndrome; and in the COSMIC database was described to be associated with large intestine, breast, esophagus, skin and stomach cancer. The most frequent variant was the missense c. $215 \mathrm{C}>\mathrm{G}$ variant, found in 17 cases of CGC (6 ALT and 11 HET) and 29 EOGC cases (17 ALT and $12 \mathrm{HET}$ ), and was also present in patients with hereditary cancer predisposing syndrome and Li-Fraumeni syndrome. Based on the COSMIC database, it was found in the large intestine and soft tissue cancers, but also in the stomach according to the International Cancer Genome Consortium.

Immunohistochemistry staining of CHEK2 and p-CHEK2 proteins. The analyzed group encompassed 196 patients, 89 with CGC and 107 with EOGC (Table IV). The first approach was to compare the results from immunohistochemical staining in EOGCs and CGC cases. Two categories, high and low expression, were distinguished. Differential expression in the cytoplasmic CHEK2 and nuclear-phosphorylated CHEK2 $(\mathrm{P}<0.001$ and $\mathrm{P}=0.011$, respectively) was statistically significant between the analyzed subgroups. The high expression of CHEK2 in the cytoplasm was predominant in the CGC subtype, where it was observed in $63 \%$ of cases. Phospho-CHEK2 was highly expressed in the nuclei of CGC in 53\%, whereas in EOGC, it was observed in only $34 \%$ of samples. The differences in the nuclear CHEK2 (CHEK2-nucleus) and cytoplasmic phosphorylated CHEK2 (phospho-CHEK2cytoplasmic) expression patterns between CGC and EOGC were not significant $(\mathrm{P}=0.178$ and $\mathrm{P}=0.133$ respectively).

Based on the result that nuclear CHEK2 expression was upregulated in both subtypes of GC, this parameter was used to distinguish a subgroup of samples with high nuclear CHEK2 expression, the 'CHEK2-nucleus high subgroup', and compared with the other parameters (Table V). In the CHEK2-nucleus high subgroup, there was a statistically significant difference 
Table IV. Comparison of nuclear and cytoplasmic CHEK2 and p-CHEK2 expression in CGC ( $\mathrm{n}=89)$ and EOGC $(\mathrm{n}=107)$ samples.

\begin{tabular}{lccr}
\hline Expression & EOGC, $\mathrm{n}(\%)$ & CGC, $\mathrm{n}(\%)$ & P-value \\
\hline CHEK2-nucleus high & $93(87)$ & $70(79)$ & 0.178 \\
CHEK2-nucleus low & $14(13)$ & $19(21)$ & $<0.001^{\mathrm{a}}$ \\
CHEK2-cytoplasmic high & $39(36)$ & $56(63)$ & $0.011^{\mathrm{a}}$ \\
CHEK2-cytoplasmic low & $68(64)$ & $33(37)$ & 0.133 \\
p-CHEK2-nucleus high & $36(34)$ & $47(53)$ & \\
p-CHEK2-nucleus low & $71(66)$ & $34(38)$ & $55(62)$ \\
p-CHEK2-cytoplasmic high & $29(27)$ & $78(73)$ &
\end{tabular}

${ }^{\mathrm{a}} \mathrm{P}<0.05$. p-CHEK2, phosphorylated checkpoint kinase 2; GC, gastric cancer; EOGC, early-onset GC; CGC, conventional GC.

Table V. Comparison of CHEK2 and p-CHEK2 expression in the nucleus and cytoplasm among the CHEK2-nucleus high subgroup between CGC $(\mathrm{n}=70)$ and EOGC $(\mathrm{n}=93)$ samples.

\begin{tabular}{lccr}
\hline Expression & $\begin{array}{c}\text { CHEK2-nucleus high } \\
\text { subgroup in EOGC, n (\%) }\end{array}$ & $\begin{array}{c}\text { CHEK2-nucleus high } \\
\text { subgroup in CGC, n (\%) }\end{array}$ & P-value \\
\hline p-CHEK2-nucleus high & $36(39)$ & $45(64)$ & $0.002^{\mathrm{a}}$ \\
p-CHEK2-nucleus low & $57(61)$ & $25(36)$ & $<0.001^{\mathrm{a}}$ \\
CHEK2-cytoplasmic high & $36(39)$ & $54(77)$ & $0.029^{\mathrm{a}}$ \\
CHEK2-cytoplasmic low & $57(61)$ & $16(23)$ & $32(46)$ \\
p-CHEK2-cytoplasmic high & $26(28)$ & $38(54)$ & \\
p-CHEK2-cytoplasmic low & $67(72)$ & & \\
\hline
\end{tabular}

${ }^{\mathrm{a}} \mathrm{P}<0.05$. p-CHEK2, phosphorylated checkpoint kinase 2; GC, gastric cancer; EOGC, early-onset GC; CGC, conventional GC.

between the expression of phospho-CHEK2 in the nucleus (phospho-CHEK2-nucleus) between CGC and ECOG $(\mathrm{P}=0.002)$; in $\mathrm{CGC}$, phospho-CHEK2 in the nucleus was upregulated in $64 \%$ of cases whereas in EOGCs it was only upregulated only in $39 \%$ cases. The levels of cytoplasmic CHEK2 expression (CHEK2-cytoplasmic) also significantly varied in the two subtypes of $\mathrm{GC}(\mathrm{P}<0.001)$. In CGC cytoplasmic CHEK2 was high in $77 \%$ of cases, whereas in EOGC it was only upregulated in $39 \%$ of the patients. Expression of phospho-CHEK2 was significantly higher in CGC compared with $\mathrm{EOGC}(\mathrm{P}=0.029)$. The remaining subgroup of low nuclear expression of CHEK2 (CHEK2-nucleus-low) was analyzed; however, none of the evaluated parameters were considered to be significantly associated.

Immunohistochemical staining of p53. The analyzed group on which p53 staining was performed consisted of 181 patients, 90 with CGC and 91 with EOGC (Table VI). Staining results for $\mathrm{p} 53$ protein in both subtypes of GC were compared. Two scoring criteria, positive and negative, were used for p53 expression assessment. Differential expression in the p53-positive and p53-negative was statistically significant between the two groups $(\mathrm{P}=0.029)$. The expression of $\mathrm{p} 53$ was higher in the CGC group, where it was observed in $49 \%$ of cases. The methodology and results of the p53 staining was previously published by Milne et al (24).
Table VI. Comparison of p53-positive and -negative expression between CGC $(n=90)$ and EOGC $(n=91)$ samples.

\begin{tabular}{lccc}
\hline p53 expression & EOGC, $n(\%)$ & CGC, $n(\%)$ & P-value \\
\hline p53-positive & $29(32)$ & $44(49)$ & $0.029^{a}$ \\
p53-negative & $62(68)$ & $46(51)$ & \\
\hline
\end{tabular}

${ }^{a} \mathrm{P}<0.05$. GC, gastric cancer; EOGC, early-onset GC; CGC, conventional GC.

The EOGC p53-positive and CGC p53-positive parameters were compared with subgroups of samples with CHEK2-nucleus and CHEK2-cytoplasmic, phosphorylated and non-phosphorylated, and high vs. low expression patterns, respectively (Table VII). There was a statistically significant difference between the expression of CHEK2-cytoplasmic high and low, and the EOGC p53-positive and CGC p53-positive patients $(\mathrm{P}=0.002)$. CHEK2-cytoplasmic was highly expressed in the CGC p53-positive group (68\%), whereas $72 \%$ of the EOGC p53-positive patients had low CHEK2-cytoplasmic expression. The remaining subgroups in Table VII were analyzed but none of the parameters were found to be significantly different between the subgroups. 
Table VII. Comparison of high and low CHEK2 and p-CHEK2 expression in the nucleus and cytoplasm among EOGC p53-positive ( $\mathrm{n}=29)$ and CGC $\mathrm{p} 53$-positive ( $\mathrm{n}=44)$ subgroups.

\begin{tabular}{lccr}
\hline Expression & EOGC p53-positive, $\mathrm{n}(\%)$ & CGC p53-positive, $\mathrm{n}(\%)$ & P-value \\
\hline CHEK2-nucleus high & $27(93)$ & $38(86)$ & 0.604 \\
CHEK2-nucleus low & $2(7)$ & $6(14)$ & $0.002^{\mathrm{a}}$ \\
CHEK2-cytoplasmic high & $8(28)$ & $30(68)$ & \\
CHEK2-cytoplasmic low & $21(72)$ & $14(32)$ & 0.149 \\
p-CHEK2-nucleus high & $10(34)$ & $24(55)$ & 0.264 \\
p-CHEK2-nucleus low & $19(66)$ & $20(45)$ & $14(32)$ \\
p-CHEK2-cytoplasmic high & $5(17)$ & $30(68)$ & \\
p-CHEK2-cytoplasmic low & $24(83)$ & $0(8)$ & \\
\hline
\end{tabular}

${ }^{\mathrm{a}} \mathrm{P}<0.05$. p-CHEK2, phosphorylated checkpoint kinase 2; GC, gastric cancer; EOGC, early-onset GC; CGC, conventional GC.

Table VIII. Comparison of high and low CHEK2 and p-CHEK2 expression in the nucleus and cytoplasm among EOGC p53-negative $(n=62)$ and CGC $p 53$-negative $(n=46)$ subgroups.

\begin{tabular}{lccc}
\hline Expression & EOGC p53-negative, n (\%) & CGC p53-negative, n (\%) & P-value \\
\hline CHEK2-nucleus high & $53(85)$ & $33(72)$ & $13(28)$ \\
CHEK2-nucleus low & $9(15)$ & $26(57)$ & 0.131 \\
CHEK2-cytoplasmic high & $21(34)$ & $20(43)$ \\
CHEK2-cytoplasmic low & $41(66)$ & $23(50)$ & $0.031^{\mathrm{a}}$ \\
p-CHEK2-nucleus high & $20(32)$ & $23(50)$ & 0.096 \\
p-CHEK2-nucleus low & $42(68)$ & $21(46)$ & $0.033^{\mathrm{a}}$ \\
p-CHEK2-cytoplasmic high & $15(24)$ & $25(54)$ &
\end{tabular}

${ }^{a} \mathrm{P}<0.05$. p-CHEK2, phosphorylated checkpoint kinase 2; GC, gastric cancer; EOGC, early-onset GC; CGC, conventional GC.

The EOGC p53-negative and CGC p53-negative subgroups were compared withCHEK2-nucleus and CHEK2-cytoplasmic, both phosphorylated and non-phosphorylated, high and low expression, respectively (Table VIII). The EOGC p53-negative and CGC p53-negative patients had a significantly different CHEK-2 cytoplasmic expression pattern $(\mathrm{P}=0.031)$. CHEK2-cytoplasmic expression was low in EOGC p53-negative patients (66\%), whereas high expression was found in $57 \%$ of CGC p53-negative patients. Phospho-CHEK2-cytoplasmic low expression was predominant in the EOGC p53-negative group (76\%), and high expression was observed in the CGC p53-negative group $(\mathrm{P}=0.033)$. The other groups in Table VIII were analyzed; however, none of the assessed parameters were statistically significant.

Correlation between the genetic alterations of CHEK2 and TP53 genes and their protein expression levels. Next, the EOGC and CGC cases were compared for the different mutation status for SNPs most frequently occurring among the analyzed groups in both genes: CHEK2 and TP53, and their protein expression levels (Table SIII). Correlations between mutations in CHEK2: c. $319+379 \mathrm{~A}>\mathrm{G}$, c. $252 \mathrm{~A}>\mathrm{G}$, c. $1246 \mathrm{~A}>\mathrm{G}$, c. $1245 \mathrm{C}>\mathrm{T}$ and CHEK2 protein expression in the nucleus and cytoplasm both in the phosphorylated and non-phosphorylated forms was compared. There was a statistically significant correlation between occurrence of the c. $319+379 \mathrm{~A}>\mathrm{G}$ alteration in both CGC and EOGC subtypes and phospho-CHEK2-nucleus expression levels $(\mathrm{P}=0.003)$. The expression of phospho-CHEK2-nucleus was higher in the CGC c. $319+379 A>G$ group ( $82 \%$ of cases). Between CGC and EOGC with two SNPs in TP53 gene: c. $215 \mathrm{C}>\mathrm{G}, \mathrm{c} .639 \mathrm{~A}>\mathrm{G}$ and p53-positive and negative staining, there was no statistically significant correlations.

\section{Discussion}

The primary function of CHEK2 is suppressing tumor growth, and exhibits proapoptotic and mitotic functions. The association between changes in CHEK2 gene expression and cancer risk development have been demonstrated in numerous case-controlled studies (34-36). The CHEK2 gene encodes a serine/threonine kinase (CHEK2), which is activated by ATM, following mobilization of the cascade of DNA damage response molecules to double-stranded breaks. Additionally, CHEK 2 is widely phosphorylated at Thr68, resulting in its activation, particularly during the development of precancerous lesions and in the progression of cancer (37). 
In the Polish population, three founder alleles were found: A missense substitution of an isoleucine for a threonine in exon 3: I157T and two alterations that truncate the CHEK2 protein: IVS2+1G $>$ A in exon 3 and 1100delC in exon 10, all of which have been previously investigated with regard to their association with predisposition to GC (16).

$\mathrm{Li}$ and Stern (38) found that prior to DNA damage, CHEK2 is associated with chromatin, and irradiation or topoisomerase inhibitors decrease this association. They observed that phospho-CHEK2 was released from chromatin following DNA damage, and accumulated in the soluble cytoplasmic and soluble nuclear fraction. CHEK2 in human cells exposed to DNA-damaging agents resulted in immediate redistribution of the activated CHEK2 throughout the nucleus, rapidly spreading the checkpoint arrest signal from localized sites of DNA damage to the soluble mobile proteins, such as Cdc25 or p53 (34). Ćmielová et al (39) showed increased levels of phospho-CHEK2 in the cytoplasmic fraction after 24 and $48 \mathrm{~h}$ of mitoxantrone treatment. In the present study, only a weak, insignificant signal was observed in the nuclear fraction at the same time points.

The reports of the subcellular distribution of CHEK2 are inconsistent and the redistribution of CHEK2 after DNA damage is very poorly described. An improved understanding of the DNA damage response and protein distribution in the cells may assist in improving the efficiency of treatment of several types of cancer and may highlight specific novel therapeutic targets.

Sequencing results revealed 5 different SNPs within the studied group of patients. Variants c.1246A $>\mathrm{G}$ (missense variant), c. $1245 \mathrm{C}>\mathrm{T}$ (synonymous variant) and c. $252 \mathrm{~A}>\mathrm{G}$ (synonymous variant) were similarly distributed between the subgroups. Amongst the SNPs detected, 3 were HET in CGC and 2 were HET in EOGC. Interestingly, the intron variant c. $319+379 \mathrm{~A}>\mathrm{G}$ was present in a high number of cases; $94 \%$ of CGCs and $86 \%$ EOGCs cases. Only variant c.252A>G has been previously shown to be associated with several types of cancer, when searching NCBI ClinVar database. However, in reference to GC, all the listed SNPs seem to be very rare and thus far, have not been broadly described. Interestingly, the intron variant c. $319+379 \mathrm{~A}>\mathrm{G}$, detected in the present study, was previously shown to be associated with esophageal squamous cell carcinoma risk, as reported by Li et al (40).

The tumor suppressor gene TP53 is one of the most regularly mutated genes in human cancers, and TP53 alterations are present in $\sim 77 \%$ of stomach cancers (41). Mutations of TP53 have been detected in the early stages of gastric carcinoma, and their frequency increases as the tumor progressed (42). TP53 has been termed 'the guardian of the genome' (41). Genomic instability causes genetic variability and accelerates the rate at which a cell acquires and accumulates mutations, which may underlie the initiation of tumorigenesis (43). In the present study, 9 different genetic alterations of the TP53 gene were detected. Most of these occurred sporadically; however, variant c. $215 \mathrm{C}>\mathrm{G}$ was present in 17 cases of CGC (6 ALT and 11 HET) and 29 cases of EOGC cases (17 ALT and 12 HET), whereas variant c.639A $>\mathrm{G}$ was present in $3 \mathrm{CGC}$ cases and 1 EOGC cases (all HET). The clinical significance of the detected variants was assessed using dbSNP and ClinVar. Most of these were associated with the Li-Fraumeni syndrome

\section{Early-onset gastric cancer}

\author{
p53-positive \\ -CHEK2-cytoplasmic low \\ p53-negative \\ -CHEK2-cytoplasmic low \\ -p-CHEK2-cytoplasmic low
}

\section{Conventional gastric cancer}

\author{
p53-positive \\ -CHEK2-cytoplasmic high \\ p53-negative \\ -CHEK2-cytoplasmic high \\ -p-CHEK2-cytoplasmic high
}

Figure 2. Differences between early-onset gastric cancer and conventional gastric cancer with respect to CHEK2 and p53 staining. p-CHEK2, phosphorylated checkpoint kinase 2 .

and hereditary cancer predisposing syndrome. According to COSMIC, five of the detected variants were previously shown to be associated with stomach cancer $(\mathrm{c} .844 \mathrm{C}>\mathrm{G}, \mathrm{c} .818 \mathrm{G}>\mathrm{A}$, c. $734 \mathrm{G}>\mathrm{A}$, c. $586 \mathrm{C}>\mathrm{T}$ and c. $215 \mathrm{C}>\mathrm{G}$ ). In the present study, it was demonstrated that the TP53 mutations may accumulate over a very long period of time (lifetime) (44). With aging, genetic damage may increase, increasing the importance of the function of TP53, and therefore making an individual more vulnerable to loss of function mutations.

Immunohistochemistry analysis may have additional value for the assessment of the $A T M>C H E K 2>p 53$ pathway. When CHEK2 is phosphorylated at Thr68, it is activated. Therefore, expression of the phosphorylated protein may be used as a potential marker of active CHEK2 status (45). The constitutive activation of the DNA damage checkpoint pathway may be associated with an increased level of p53 alterations in cancer, taking into consideration that p53 is a downstream target of ATM and CHEK2. The immunohistochemical analyses performed in the present study included analysis of cytoplasmic and nuclear expression of CHEK2 and phospho-CHEK2.

The levels and distribution of expression of these proteins appeared to vary between CGC and EOGC. Interestingly, the levels of p-CHEK2 increased with age, and a statistical difference between the two subtypes with regard to nuclear 
p-CHEK2 expression was observed. Additionally, high nuclear CHEK2 expression was also more prevalent in CGC, following stratification based on levels of nuclear CHEK2 expression. Cytoplasmic phospho-CHEK2 expression was increased. The phosphorylated forms of both CHEK2 types may be an indicator of $\mathrm{GC}$ development. The levels of CHEK2 protein in the nuclei was elevated in both subtypes of GC, and this outcome supports further study into the potential therapeutic value of CHEK2.

p53 staining was performed on 181 tumor samples, in both age dependent subtypes of GC; expression of the p53 protein increased with age. Immunohistochemistry analysis of p53 may explain the biology of the tumor. Multiple studies have used immunohistochemistry analysis to assess p53 expression. In lung cancer, a meta-analysis showed that positive immunohistochemistry staining of p53 may be used as a prognostic biomarker (46). The results of the present study showed that p53 expression could be used to distinguish age dependent onset of GC.

The EOGC p53-positive and CGC p53-positive groups were compared based on phosphorylation of CHEK2. There was a statistically significant difference between the expression of CHEK2-cytoplasmic high and low, and the EOGC p53-positive and CGC p53-positive patients. The expression of CHEK2 in the cytoplasm was increased (68\%) in the CGC p53-positive group. Statistically important differences between CHEK2-cytoplasmic high and low, and the EOGC and CGC p53-negative patients were also observed. CHEK2-cytoplasmic expression was increased in CGC p53-negative patients (57\%), and its expression was higher in the CGC p53-positive subgroup (68\%). Phospho-CHEK2-cytoplasmic was also higher amongst the CGC TP53-negative group. Together, these results suggests a link between the expression of p53 and CHEK2 cytoplasmic proteins in GC development based on age. Fig. 2 summarizes the primary differences between EOGC and CGC with respect to CHEK2 and p53 staining.

Non-coding regions possess regulatory functions for the coding regions. Thus coding regions are the primary actors, but their function is regulated by non-coding regions. Thus any modifications to the non-coding may have an impact on the expression and regulation of the expressed genes. There was a statistically significant correlation between occurrence of the c.319+379A $>\mathrm{G}$ intronic alteration in both $\mathrm{CGC}$ and EOGC subtypes and phospho-CHEK2-nuclear expression, which increased with age.

The present study has some limitations. First, the number of biopsies from patients was a limiting factor. The selected subgroups were small, and thus it is difficult to observe the differences in SNP frequencies in the CHEK2 gene. Use of a benign gastric disease control group may better allow for determination of whether the protein expression of these two genes may be used as biomarkers of early gastric cancer development. In vitro studies are required to determine the functional effects of the detected SNPs in more detail. It is also probable that different allele frequency amongst individuals of different ethnicities may have an impact on the obtained data. Thus, a more diverse cohort may allow for identification of differences in frequencies of SNPs in different populations around the world.

\section{Acknowledgements}

Not applicable.

\section{Funding}

The present study was partly supported by a grant from the Polish Ministry of Science and Higher Education (grant no. NN402423838).

\section{Availability of data and materials}

The NGS datasets generated and/or analyzed during the current study are available in the Open Science Framework Repository. Outcome for the 94 genes and 284 SNPs presented in the NGS panel are available at https://osf.io/9483c/(snps.variants-all.csv) and https://osf.io/zxwg9/(genes.variants-all.csv).

\section{Authors' contributions}

PW and RS conceptualized the study. JM, FM, GJAO and RS developed the methodology. PK and PW performed the data processing. JM, PK, AB and RS analyzed the results. JM, PK, MS, AB, FM, PW, RM, GJAO and RS performed the experiments. PW and RS confirmed the authenticity of the data. JM, MS, AB and RS wrote the original draft. JM, PK, MS, AB, FM, PW, RM, GJAO and RS reviewed and edited the manuscript. PW, GJAO and RS supervised the study. RM and RS acquired funding. All authors read and approved the final manuscript.

\section{Ethics approval and consent to participate}

All procedures involving human participants were in accordance with the ethical standards of the Medical University of Lublin Bioethical Committee (Lublin, Poland; approval no. KE-0254/322/2019) and with the 1964 Declaration of Helsinki and its later amendments or comparable ethical standards. Due to the retrospective nature of the study, patient consent was waived by the ethics committee.

\section{Patient consent for publication}

Not applicable.

\section{Competing interests}

The authors declare that they have no competing interests.

\section{References}

1. Zou Z and Jemal A: Cancer statistics, 2014. CA Cancer J Clin 64: 9-29, 2014.

2. Howlader N, Noone AM, Krapcho M, Neyman N, Aminou R, Waldron W, Altekruse SF, Kosary CL, Ruhl J, Tatalovich Z, et al: SEER Cancer Statistics Review, 1975-2008, National Cancer Institute. Bethesda, MD, pp19, 2011.

3. Milne AN, Sitarz R, Carvalho R, Carneiro F and Offerhaus GJ: Early onset gastric cancer: On the road to unraveling gastric carcinogenesis. Curr Mol Med 7: 15-28, 2007.

4. Milne AN, Carneiro F, O'Morain C and Offerhaus GJ: Nature meets nurture: Molecular genetics of gastric cancer. Hum Genet 126: 615-628, 2009. 
5. Milne AN, Offerhaus GJ and Carneiro F: Histopathology of familial and early-onset gastric cancer. Diagnostic Histopathol 17: 62-68, 2011

6. Slavin T, Neuhausen SL, Rybak C, Solomon I, Nehoray B, Blazer K, Niell-Swiller M, Adamson AW, Yuan YC, Yang K, et al: Genetic gastric cancer susceptibility in the international clinical cancer genomics community research network. Cancer Genet 216-217: 111-119, 2017.

7. Cybulski C, Górski B, Huzarski T, Masojć B, Mierzejewski M, Debniak T, Teodorczyk U, Byrski T, Gronwald J, Matyjasik J, et al: CHEK2 is a multiorgan cancer susceptibility gene. Am J Hum Genet 75: 1131-1135, 2004.

8. Al-Rakan MA, Hendrayani SF and Aboussekhra A: CHEK2 represses breast stromal fibroblasts and their paracrine tumor-promoting effects through suppressing SDF-1 and IL-6. BMC Cancer 16: 575, 2016.

9. Desrichard A, Bidet Y, Uhrhammer N and Bignon YJ: CHEK2 contribution to hereditary breast cancer in non-BRCA families. Breast Cancer Res 13: R119, 2011.

10. Wang N, Ding H, Liu C, Li X, Wei L, Yu J, Liu M, Ying M, Gao W, Jiang H and Wang Y: A novel recurrent CHEK2 Y390C mutation identified in high-risk Chinese breast cancer patients impairs its activity and is associated with increased breast cancer risk. Oncogene 34: 5198-5205, 2015

11. Dong X, Wang L, Taniguchi K, Wang X, Cunningham JM, McDonnell SK, Qian C, Marks AF, Slager SL, Peterson BJ, et al: Mutations in CHEK2 associated with prostate cancer risk. Am J Hum Genet 72: 270-280, 2003.

12. Hale V, Weischer M and Park JY: CHEK2 (*) 1100delC mutation and risk of prostate cancer. Prostate Cancer 2014: 294575, 2014.

13. Cybulski C, Masojc B, Oszutowska D, Jaworowska E, Grodzki T, Waloszczyk P, Serwatowski P, Pankowski J, Huzarski T, Byrski T, et al: Constitutional CHEK2 mutations are associated with a decreased risk of lung and laryngeal cancers. Carcinogenesis 29: 762-765, 2008

14. Kilpivaara O, Alhopuro P, Vahteristo $\mathrm{P}$, Aaltonen LA and Nevanlinna H: CHEK2 I157T associates with familial and sporadic colorectal cancer. J Med Genet 43: e34, 2006.

15. Skierucha M, Milne AN, Offerhaus GJA, Polkowski WP, Maciejewski R and Sitarz R: Molecular alterations in gastric cancer with special reference to the early-onset subtype. World J Gastroenterol 22: 2460-2474, 2016.

16. Teodorczyk U, Cybulski C, Wokołorczyk D, Jakubowska A, Starzyńska T, Lawniczak M, Domagała P, Ferenc K, Marlicz K, Banaszkiewicz Z, et al: The risk of gastric cancer in carriers of CHEK2 mutations. Fam Cancer 12: 473-478, 2013.

17. Buscemi G, Carlessi L, Zannini L, Lisanti S, Fontanella E, Canevari S and Delia D: DNA damage-induced cell cycle regulation and function of novel Chk2 phosphoresidues. Mol Cell Biol 26: 7832-7845, 20060.

18. McBride OW, Merry D and Givol D: The gene for human p53 cellular tumor antigen is located on chromosome 17 short arm (17p13). Proc Natl Acad Sci USA 83: 130-134, 1986

19. Barnoud T, Parris JLD and Murphy ME: Common genetic variants in the TP53 pathway and their impact on cancer. J Mo Cell Biol 11: 578-585, 2019.

20. Fenoglio-Preiser CM, Wang J, Stemmermann GN and Noffsinger A: TP53 and gastric carcinoma: A review. Hum Mutat 21: 258-270, 2003

21. Blondal JA and Benchimol S: The role of p53 in tumor progression. Semin Cancer Biol 5: 177-186, 1994.

22. Lakin ND and Jackson SP: Regulation of p53 in response to DNA damage. Oncogene 18: 7644-7655, 1999.

23. Laurén P: The two histological main types of gastric carcinoma: Diffuse and so-called intestinal-type carcinoma. An attempt at a histo-clinical classification. Acta Pathol Microbiol Scand 64 31-49, 1965.

24. Milne AN, Carvalho R, Morsink FM, Musler AR, de Leng WW, Ristimäki A and Offerhaus GJ: Early-onset gastric cancers have a different molecular expression profile than conventional gastric cancers. Mod Pathol 19: 564-572, 2006.

25. Sitarz R, Leguit RJ, de Leng WW, Polak M, Morsink FM, Bakker O, Maciejewski R, Offerhaus GJ and Milne AN: The COX-2 promoter polymorphism-765 $\mathrm{G}>\mathrm{C}$ is associated with early-onset, conventional and stump gastric cancers. Mod Pathol 21: 685-690, 2008

26. Li H, Handsaker B, Wysoker A, Fennell T, Ruan J, Homer N, Marth G, Abecasis G and Durbin R; 1000 Genome Project Data Processing Subgroup: The sequence Alignment/Map format and SAMtools. Bioinformatics 25: 2078-2079, 2009.
27. McKenna A, Hanna M, Banks E, Sivachenko A, Cibulskis K, Kernytsky A, Garimella K, Altshuler D, Gabriel S, Daly M and DePristo MA: The genome analysis toolkit: A MapReduce framework for analyzing next-generation DNA sequencing data. Genome Res 20: 1297-1303, 2010.

28. Narzisi G, O'Rawe JA, Iossifov I, Fang H, Lee YH, Wang Z, Wu Y, Lyon GJ, Wigler M and Schatz MC: Accurate de novo and transmitted indel detection in exome-capture data using microassembly. Nat Methods 11: 1033-1036, 2014.

29. Cingolani P, Platts A, Wang le L, Coon M, Nguyen T, Wang L, Land SJ, Lu X and Ruden DM: A program for annotating and predicting the effects of single nucleotide polymorphisms, SnpEff: SNPs in the genome of Drosophila melanogaster strain w1118; iso-2; iso-3. Fly (Austin) 6: 80-92, 2012.

30. Rugge M, Shiao YH, Busatto G, Cassaro M, Strobbe C, Russo VM, Leo G, Parenti AR, Scapinello A, Arslan P and Egarter-Vigl E: The p53 gene in patients under the age of 40 with gastric cancer: Mutation rates are low but are associated with a cardiac location. Mol Pathol 53: 207-210, 2000.

31. Xu JH, Chen LR, Wang HJ and Yao LF: Application of immunohistochemistry-Laser microdissection-PCR technique in detecting p53 gene mutation in paraffin sections of gastric cancer. Zhonghua Bing Li Xue Za Zhi 33: 342-345, 2004 (In Chinese)

32. Gleeson CM, Sloan JM, McManus DT, Maxwell P, Arthur K, McGuigan JA, Ritchie AJ and Russell SE: Comparison of p53 and DNA content abnormalities in adenocarcinoma of the oesophagus and gastric cardia. Br J Cancer 77: 277-286, 1998.

33. Kim SS, Bhang CS, Min KO, Chae HS, Choi SW, Lee CD, Lim KW, Chung IS and Park DH: p53 mutations and microsatellite instabilities in the subtype of intestinal metaplasia of the stomach. J Korean Med Sci 17: 490-496, 2002.

34. Bartek J and Lukas J: Chk1 and Chk2 kinases in checkpoint control and cancer. Cancer Cell 3: 421-429, 2003.

35. Cybulski C, Wokołorczyk D, Jakubowska A, Huzarski T, Byrski T, Gronwald J, Masojé B, Deebniak T, Górski B, Blecharz P, et al: Risk of breast cancer in women with a CHEK2 mutation with and without a family history of breast cancer J Clin Oncol 29: 3747-3752, 2011

36. Siołek M, Cybulski C, Gasior-Perczak D, Kowalik A, Kozak-Klonowska B, Kowalska A, Chłopek M, Kluźniak W, Wokołorczyk D, Pałyga I, et al: CHEK2 mutations and the risk of papillary thyroid cancer. Int J Cancer 137: 548-552, 2015.

37. Zoppoli G, Solier S, Reinhold WC, Liu H, Connelly JW Jr, Monks A, Shoemaker RH, Abaan OD, Davis SR, Meltzer PS, et al: CHEK2 genomic and proteomic analyses reveal genetic inactivation or endogenous activation across the 60 cell lines of the US National Cancer Institute. Oncogene 31: 403-418, 2012.

38. Li J and Stern DF: DNA damage regulates Chk2 association with chromatin. J Biol Chem 280: 37948-37956, 2005.

39. Ćmielová J, Lesná M and Řezáčová M: Subcellular localization of proteins responding to mitoxantrone-induced DNA damage in leukaemic cells. Folia Biol (Praha) 61: 60-65, 2015.

40. Li WQ, Hu N, Hyland PL, Gao Y, Wang ZM, Yu K, Su H, Wang CY, Wang LM, Chanock SJ, et al: Genetic variants in DNA repair pathway genes and risk of esophageal squamous cell carcinoma and gastric adenocarcinoma in a Chinese population. Carcinogenesis 34: 1536-1542, 2013.

41. Lane DP: Cancer. p53, guardian of the genome. Nature 358: $15-16,1992$

42. Vousden KH and Prives C: P53 and prognosis: New insights and further complexity. Cell 120: 7-10, 2005.

43. Hanahan D and Weinberg RA: Hallmarks of cancer: The next generation. Cell 144: 646-674, 2011.

44. Feng Z, Hu W, Teresky AK, Hernando E, Cordon-Cardo C and Levine AJ: Declining p53 function in the aging process: A possible mechanism for the increased tumor incidence in older populations. Proc Natl Acad Sci USA 104: 16633-16638, 2007.

45. DiTullio RA Jr, Mochan TA, Venere M, Bartkova J, Sehested M, Bartek J and Halazonetis TD: 53BP1 functions in an ATM-dependent checkpoint pathway that is constitutively activated in human cancer. Nat Cell Biol 4: 998-1002, 2002.

46. Steels E, Paesmans M, Berghmans T, Branle F, Lemaitre F, Mascaux C, Meert AP, Vallot F, Lafitte JJ and Sculier JP: Role of p53 as a prognostic factor for survival in lung cancer: A systematic review of the literature with a meta-analysis. Eur Respir J 18: 705-719, 2001.

c) (i) $\odot$ This work is licensed under a Creative Commons Attribution-NonCommercial-NoDerivatives 4.0 International (CC BY-NC-ND 4.0) License. 